\title{
Introduction to the fast track issue for CPAIOR 2015
}

\author{
L. Michel $^{1}$
}

Published online: 28 April 2015

(C) Springer Science+Business Media New York 2015

The purpose of the CPAIOR Conference is to promote collaboration among researchers from Constraint Programming, Artificial Intelligence and Operations Research to produce cutting-edge research results that integrate advances from all three fields and deliver solution techniques for complex and challenging problems in the combinatorial optimization space. Papers presented at the conference undergo a rigorous review process to yield a technical program that is diverse, compelling, and relevant to practitioners as it also reports on applications whose resolution showcase hybrid techniques.

The $12^{\text {th }}$ edition of the International Conference was held on May 18-22, 2015 in Barcelona, Spain. The call for papers solicited submissions covering but not limited to the following topics:

Inference and relaxation methods: constraint propagation, cutting planes, global constraints, graph algorithms, dynamic programming, Lagrangian and convex relaxations and heuristic functions based on constraint relaxation.

Search methods: branch and bound, incomplete search, intelligent backtracking, randomized search, portfolios, column generation, Benders decompositions or any other decomposition methods, local search, meta-heuristics.

Integration methods: solver communication, model transformations and solver selection, parallel and distributed resolution techniques, models, and solvers.

Modeling methods: comparison of models, symmetry breaking, uncertainty, dominance relationships.

Applications: innovative Applications of CPAIOR techniques.

Implementation: implementation of CPAIOR techniques and optimization systems.

The call for paper also advertised a Journal fast track as a vehicle to route outstanding papers directly to the Constraint Journal. During the review process, the program committee

L. Michel

ldm@engr.uconn.edu

1 Computer Science, Engineering Department, University of Connecticut,

371 Fairfield Road, Storrs, CT 06269-4155, USA 
identified remarkable submissions and recommend them to the journal fast track. Authors of these papers were given the option to pursue this opportunity with an extended submission and additional rounds of review on an accelerated schedule. This issue of the constraint journal collects the selected five papers.

In A Column-Generation Approach for Joint Mobilization and Evacuation Planning, Pillac et al. consider large scale evacuation problems for natural disaster. Their approach focuses, for the first time, on the ability to simultaneously address both the mobilization and evacuation planning aspects while taking into account the response from evacuees. The solution methodology leverages a column generation approach to maximize the number of evacuees while minimizing the actual duration of the operation.

In A hybrid exact method for a scheduling problem with a continuous resource and energy constraints, Nattaf et al. consider a scheduling problem blending continuous resource usage with energy constraints. In this generalization of cumulative scheduling, the objective is to find a feasible schedule. The paper develops a mixed Integer Linear Program based on an event-driven formulation and builds to a hybrid branch-and-bound method to solve the problem.

In A Constraint-Based Local Search Backend for MiniZinc, Björdal et al. explore an extension to the MiniZinc modeling language meant to support Constraint-Based Local Search. The paper discusses the various challenges that arise in the process of designing this extension while offering a prototype based on the Constraint-Based Local Search Engine found in OscaR.

In Lagrangian Bounds from Decision Diagrams, Bergman et al. investigate the expansion of Decision Diagrams to incorporate side constraints through a Lagrangian relaxation directly embedded within the objective function modeled by the decision diagram. The paper explores the modeling and resolution and shares empirical results on a sample problem: a traveling salesman with time windows.

Finally, in New filtering for ATMOSTNVALUE and its weighted variant: A Lagrangian approach, Cambazard and Fages re-examine the ATMOSTNVALUE global constraint and its weighted counterpart and offer filtering algorithms based on Lagrangian relaxations. Their investigation establishes that the Lagrangian relaxation embedded within the filtering algorithms can deliver significant benefits over established techniques and highlights the potential of Lagrangian relaxation within Constraint Programming frameworks.

I wish to express my deepest gratitude to the authors an the reviewers. Their commitment to a tight schedule allowed us to complete this issue within the allotted time. Finally, I am particularly thankful to Michela Milano, Editor-in-chief for Constraints who gave the idea of a journal fast track and provided us with the opportunity to create this issue.

Laurent D. Michel, Storrs, April 2015 